\title{
MODELAGEM DE NANOBIOSSENSOR NÃO INTRUSIVO PARA DETECÇÃO PRECOCE DO CÂNCER DE MAMA ${ }^{1}$
}

\author{
MODELLING A NON-INTRUSIVE NANOBIOSENSOR FOR \\ BREAST CANCER EARLY DIAGNOSIS
}

\author{
Júlia Vaz Schultz ${ }^{2}$ e Mirkos Ortiz Martins ${ }^{3}$
}

\section{RESUMO}

O câncer de mama é a $2^{\mathrm{a}}$ doença de ocorrência mais frequente no mundo, e seu diagnóstico é comumente estabelecido quando a doença já está em estágio avançado, reduzindo a elaboração de estratégias de tratamento e as possibilidades de cura. Adicionalmente, os métodos de diagnóstico utilizados baseiam-se em exames de imagem e biópsia, intrusivos e desconfortáveis ao paciente. Neste cenário, a nanotecnologia mostra seu potencial na área de diagnósticos, principalmente com a criação de nanobiossensores, dispositivos que permitem o diagnóstico precoce de doenças por meio de análises a nível molecular. Este trabalho demonstra a modelagem de um sistema de detecção da proteína HER2, a qual pode ser encontrada tanto em amostras teciduais, no sangue, e saliva. Os sistemas propostos para reconhecimento da proteína HER2 foram baseados no Óxido de Grafeno (GO) associado a dois tipos de moléculas: biotina (BTN) e etileno glicol (EDO), ligados por dock ao anticorpo monoclonal humanizado Anti-HER2. Foram utilizados softwares de modelagem molecular para construção e análise das estruturas, gerando como resultados a visualização da conformação tridimensional formada pelos sistemas e medição das energias de interação intermoleculares resultantes, possibilitando a escolha do melhor sistema para deteç̧ão do HER2, o sistema GO+BTN com Anti-HER2.

Palavras-chave: HER 2, biomarcador tumoral, nanotecnologia computacional, docking.

\section{ABSTRACT}

Breast cancer ist the second more frequently occur disease, and furthermore it is commonly later diagnosed mostly in advanced stages, reducing the treatment strategies and cure possibility. The actual diagnosis methods are image based systems and biopsy, which are intrusive and uncomfortable for the patient. In this sense, the nanotechnology shows they potencial for the creation of nanobiosensor, dispositives allowing early diagnosis through molecular analysis. This work shows the modelling of a system that detects the HER2 protein, which can be found in tissues, blood and even in saliva. These Graphene Oxide (GO) based proposed recognizing systems is associated with two different molecules: biotin (BTN) and ethylene glycol (EDO), linked by dock on the monoclonal humanized antibody Anti-HER2 Molecular modelling softwares where applied to generate and analyze the structures, getting results of the tridimensional conformation formed in the systems and the values of intermolecular energy, which allows to choose the best GO+BTN and Anti-HER2 system to detect the HER2 protein.

Keywords: HER 2, tumoral biomarker, computational nanotechnology, docking.

\footnotetext{
${ }^{1}$ Trabalho de Iniciação Científica.

${ }^{2}$ Acadêmica do Curso de Engenharia Biomédica - Universidade Franciscana - UFN. E-mail: julia.schultz@ufn.edu.br

${ }^{3}$ Professor do curso de Engenharia Biomédica, Orientador - Universidade Franciscana - UFN. E-mail: mirkos@ufn.br
} 


\section{INTRODUÇÃO}

$\mathrm{O}$ câncer de mama é a $2^{\mathrm{a}}$ doença mais frequente desenvolvida pelas mulheres, e representa 29,5\% dos novos casos de câncer em mulheres no Brasil, de acordo com o Ministério da Saúde (2019, seç. Estatísticas de Câncer). Essa doença tem início a partir da modificação célular e posterior proliferação acelerada destas células anormalas, formando massas tumorais que podem ser classificadas como malignas. A agressividade da doença e complexidade dos tratamentos aumentam de acordo com o estágio no qual a doença se apresenta no organismo (MINISTÉRIO DA SAÚDE, 2019).

Classifica-se o avanço da doença em quatro estágios, desde o aparecimento de modificações nas células até a formação de massa tumoral, seguido pela proliferação da doença para além de seu local primário de incidência. No caso do câncer de mama, a maioria dos casos é descoberto pelas próprias mulheres quando o tumor já apresenta tamanho significativo para ser palpável, ou seja, quando em estágio avançado o suficiente para formar massa tumoral. Nestas circunstâncias as possibilidades de tratamento reduzem, bem como podem apresentar menor eficácia (MINISTÉRIO DA SAÚDE, 2019, seç. Câncer de mama: Vamos falar sobre isso?). Desta forma, os testes palpatórios, realizados pelas próprias mulheres, constituem uma ferramenta importante, entretanto de eficácia discutível para o diagnóstico precoce.

Além disso, o principal exame para diagnóstico médico é a mamografia, onde é possível a detecção de microcalcificações nas mamas. O diagnóstico médico é realizado de acordo com o fluxograma 1. Após a mamografia, quando a formação de massa anormal é detectada, a paciente deve ser submetida à biópsia, onde é retirada uma parte dessa massa para análise em laboratório, com a utilização de uma agulha de pulsão. Essa amostra é analisada para definir se o tumor é benigno ou maligno. Quando a malignidade do tumor é confirmada, ainda é feito um teste chamado de Hibridação Fluorescente In Situ (FISH), para definição molecular, onde o tumor pode ser classificado em Luminal A, Luminal B, HER2+ (HER2 positivo), triplo negativo, entre outros. Estes exames classificam o tumor e permitem aferições preditivas da doença, assim, são essenciais para decisão do método de tratamento adequado (DELMONICO; ALVES; DO AMARAL, 2015).

No entanto, a mamografia apresenta falso resultado em 15\% dos casos (SALAHANDISH et al., 2018). Esse exame ainda pode trazer riscos, como: introdução a tratamentos desnecessários quando o resultado é falso positivo, o atraso do tratamento quando o resultado é falso negativo, e o elevado risco de incidência da doença devido à frequente exposição a raios-X (GØTZSCHE; JØRGENSEN, 2013).

Neste cenário, a nanotecnologia apresenta-se como um campo de novas possibilidades, a fim de possibilitar novos métodos de diagnóstico, principalmente com o desenvolvimento de nanobiossensores. Um nanobiossensor é um dispositivo capaz de detectar biomarcadores em pequenas quantidades amostrais e assim permite diagnóstico rápido, não intrusivo e confiável. O funcionamento desses dispositivos baseia-se na interação das moléculas biomarcadoras com um biorreceptor, elemento 
do nanobiossensor, que deve apresentar tendência a se ligar ao biomarcador, pois a partir da interação destas estruturas o dispositivo é capaz de oferecer uma resposta ao sistema (PARREIRA, 2010).

Figura 1 - Representação do método tradicional de diagnóstico do câncer de mama.

\section{MÉTODO TRADICIONAL}

1'PASSO: MAMOGRAFIA

Indica a presença de calcificações nas mamas

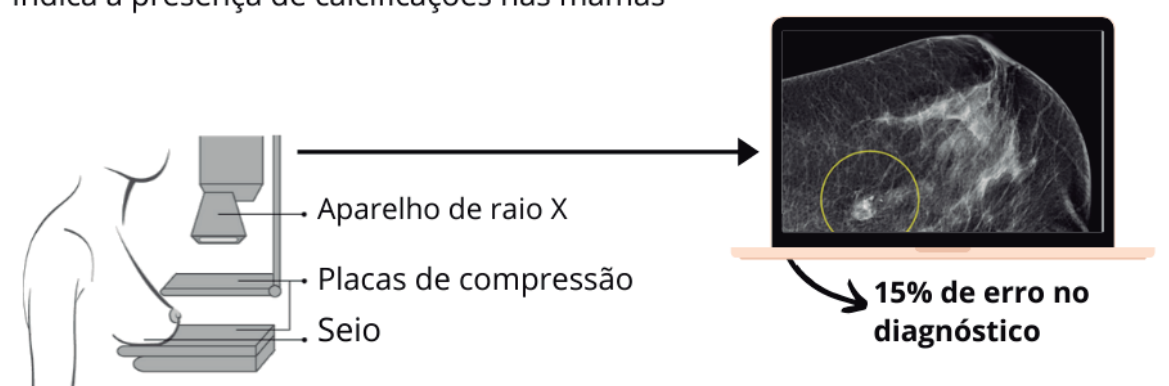

2PASSO: BIÓPSIA

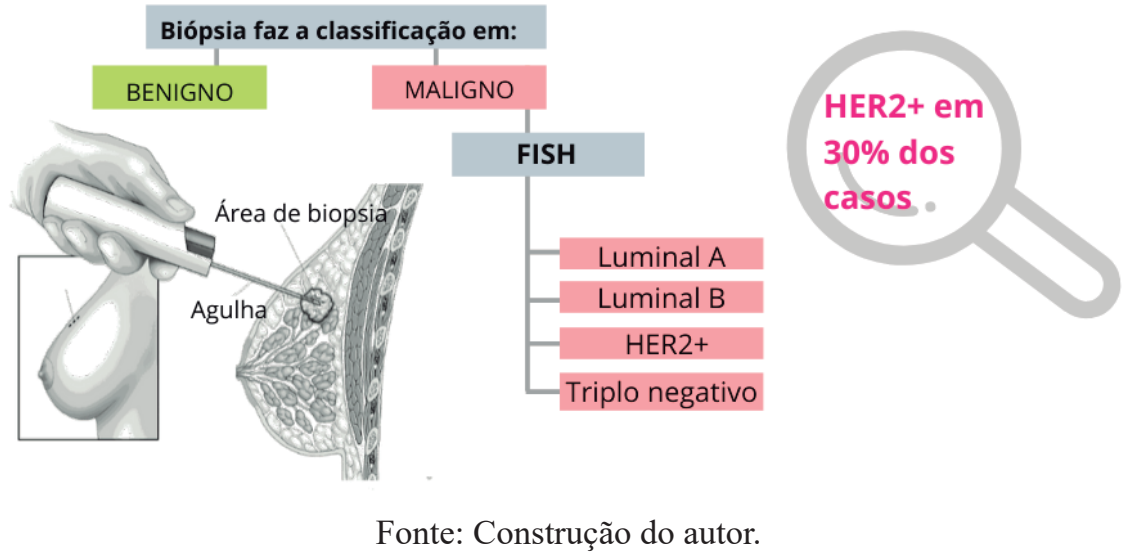

O câncer de mama apresenta alguns biomarcadores, sendo a proteína HER2 (Human Epidermal Factor Receptor type 2) o escolhido para este trabalho. Altas taxas desta proteína são encontradas no sangue de pessoas com câncer de mama HER2+, subtipo mais agressivo, até 6 meses antes do aparecimento das primeiras células tumorais modificadas (SILVA, 2015). Além disso, estudos comprovam a presença do HER2 tanto em amostras de tecido, como no sangue e saliva (CANTIGA, 2015). A detecção dos níveis de HER2 é essencial para escolha e acompanhamento do tratamento para a doença, tendo em vista que a diminuição da taxa de HER2 mostra que o tratamento está funcionando, sendo assim é considerado um biomarcador do câncer de mama.

O desenvolvimento de nanobiossensores exige, portanto, a definição de um biorreceptor, e um método de análise adequados para detectar o biomarcador da doença de interesse, o que pode ser feito com auxílio da nanotecnologia computacional, por meio da modelagem dos sistemas nanoestruturados e de suas interações entre si e com diversos meios. Dessa maneira, é possível a predição do funcionamento dos dispositivos antes mesmo de sua construção (NETO, 2014). A técnica de modelagem e simulação conhecida por docking, em especial, permite a predição de interação entre moléculas a 
partir de estruturas tridimensionais isoladas, prevendo a estrutura geométrica resultante da conformação do ligante, bem como a afinidade das interações físico-químicas (PAULA; HORTA, [s.d.]).

$\mathrm{O}$ nanobiossensor proposto será constituído por GO ligado à $\mathrm{BTN}$ ou $\mathrm{EDO}$, e funcionalizado pelo Anti-HER2 (Figura 2). Assim, a base de GO contará com uma das moléculas ligantes, e estas serão ligadas ao biorreceptor Anti-HER2, o qual deve interagir com o HER2 presente em uma amostra salivar da paciente com câncer de mama. O dispositivo assim indicará a presença da proteína HER2, sendo possível sua utilização para facilitar os métodos de diagnóstico, de modo mais rápido e menos intrusivo que os mostrados no fluxograma 1.

O presente trabalho busca modelar e simular computacionalmente, por meio da técnica docking, o funcionamento de um nanobiossensor, especificamente de seu bioreceptor, em uma base de GO que seja capaz de detectar a proteína HER2 em amostras salivares, e deverá indicar a positividade de pacientes com câncer de mama HER2+, subtipo que representa 30\% dos casos de câncer de mama. O desenvolvimento desse nanobiosensor deve ser utilizado para diagnóstico a partir de amostras salivares, garantindo assim que contribuir para que o diagnóstico de câncer seja feito de maneira mais confiável, não invasiva, e evitando que a paciente sofra qualquer desconforto e/ou consequência negativas que decorrem da submissão aos exames de imagem e biópsia.

Figura 2 - Método de diagnóstico proposto.

\section{MÉTODO ALTERNATIVO SUGERIDO}

\section{ANÁLISE DE AMOSTRA SALIVAR}

Pacientes HER2+ apresentam essa molécula na saliva.

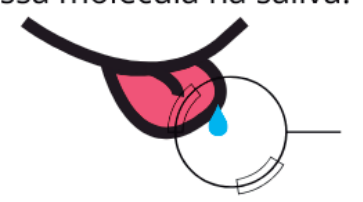

\section{Como funciona?}

A saliva é analisada por meio de um nanobiossensor, o qual detecta a presença de molécula HER2+.

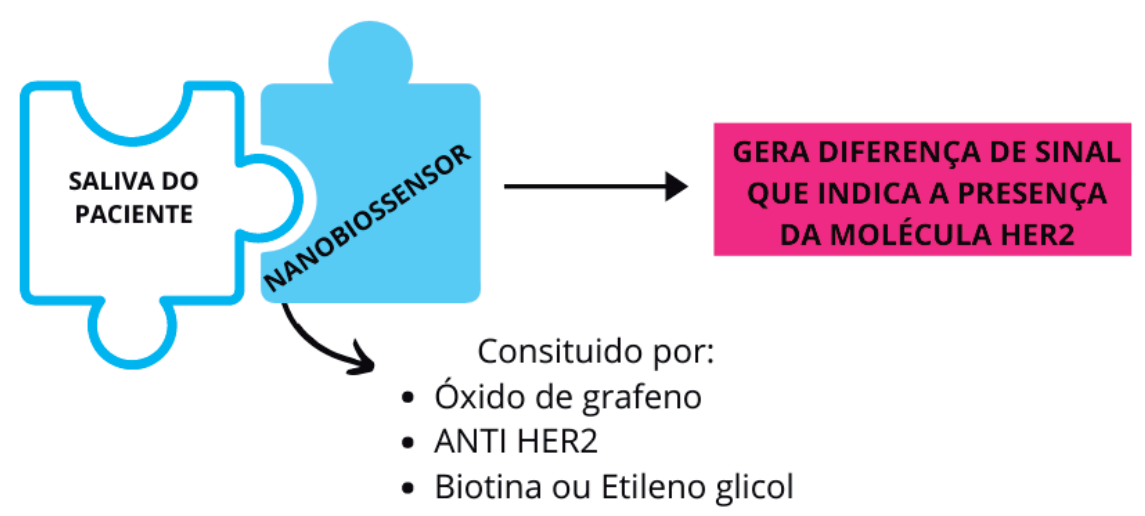

Fonte: Construção do autor. 


\section{MATERIAIS E MÉTODOS}

O sistema biorreceptor proposto para identificação da proteína HER2 (biomarcador) usa o anticorpo monoclonal humanizado anti-HER2 como biorreceptor. Este tipo de anticorpo é encontrado em medicamentos terapêuticos como o Trastuzumabe (Herceptin) (CARNEY et al., 2003). A molécula Anti-HER2, identificada sob código 6MH2 no Protein Data Bank (PDB) e cujo nome comercial é Herceptin Fab, pode ser visualizado em formato cartoon na figura 3A. Sua estrutura possui 5736 átomos e conta com duas cadeias proteicas na sua formação, resultando num peso molecular de 96.367,24 g/mols. Conforme a descrição técnica que consta no PDB, essa estrutura foi obtida experimentalmente por Difusão de vapor em diferentes trabalhos científicos (BERMAN et al., 2000, seç. Structure of Herceptin Fab without antigen).

O Anti-HER2 tem propensão à ligação com as moléculas de vitamina B7 (conhecidas por BTN) e etileno glicol (EDO), as quais também se mostram propensas à interação com GO, de acordo o Protein Data Bank (BERMAN et al., 2000, seç. Trastuzumab Fab D185A (Light Chain) Mutant Biotin Conjugation.) A estrutura da $\mathrm{BTN}$ pode ser vista na figura $3 \mathrm{~B}$, sua fórmula química é $\mathrm{C}_{10} \mathrm{H}$ ${ }_{16} \mathrm{~N}_{2} \mathrm{O}_{3} \mathrm{~S}$ e seu peso molecular de 244,311 g/mol. O EDO (figura 3C) é uma estrutura mais simples em relação a BTN; sua fórmula química é $\mathrm{C}_{2} \mathrm{H}_{6} \mathrm{O}_{2}$ e seu peso molecular é de $62,068 \mathrm{~g} / \mathrm{mol}$. A utilização dessas estruturas no sistema deve permitir melhor ligação do Anti-HER2 com o GO, e assim aumenta a confiabilidade do dispositivo.

Figura 3 - Representação tridimensional do Anti-HER2 (3A), Biotina (3B) e etilenoglicol (3C).

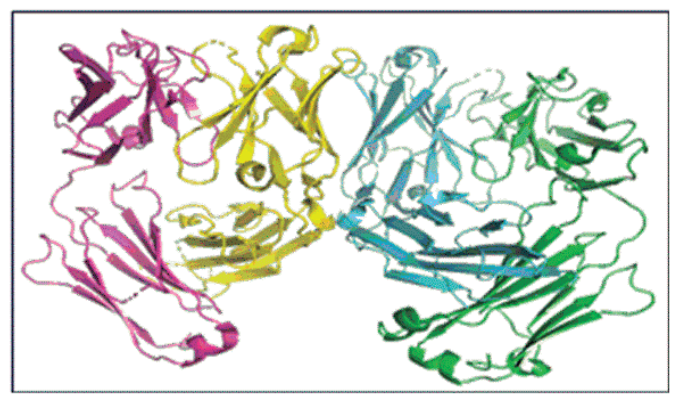

$3 \mathrm{~A}$

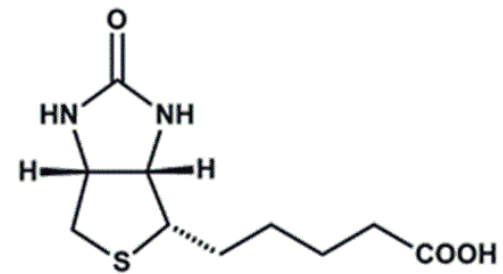

3B

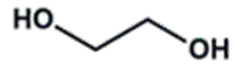

$3 \mathrm{C}$

Fonte: Construção do autor.

Anterior à construção de um nanobiossensor, os estudos em nanotecnologia computacional são utilizados. Assim, é possível a previsão do funcionamento do sistema, por meio de simulações de interação entre as moléculas (docking). Para modelagem do sistema, foi seguido o fluxograma 1. $\mathrm{O}$ docking foi feito com ambas as estruturas, sendo possível analisar a presença dos ligantes em diversos locais da estrutura do Anti-HER2. 
Fluxograma 1 - Métodos para modelagem do sistema do nanobiossensor.

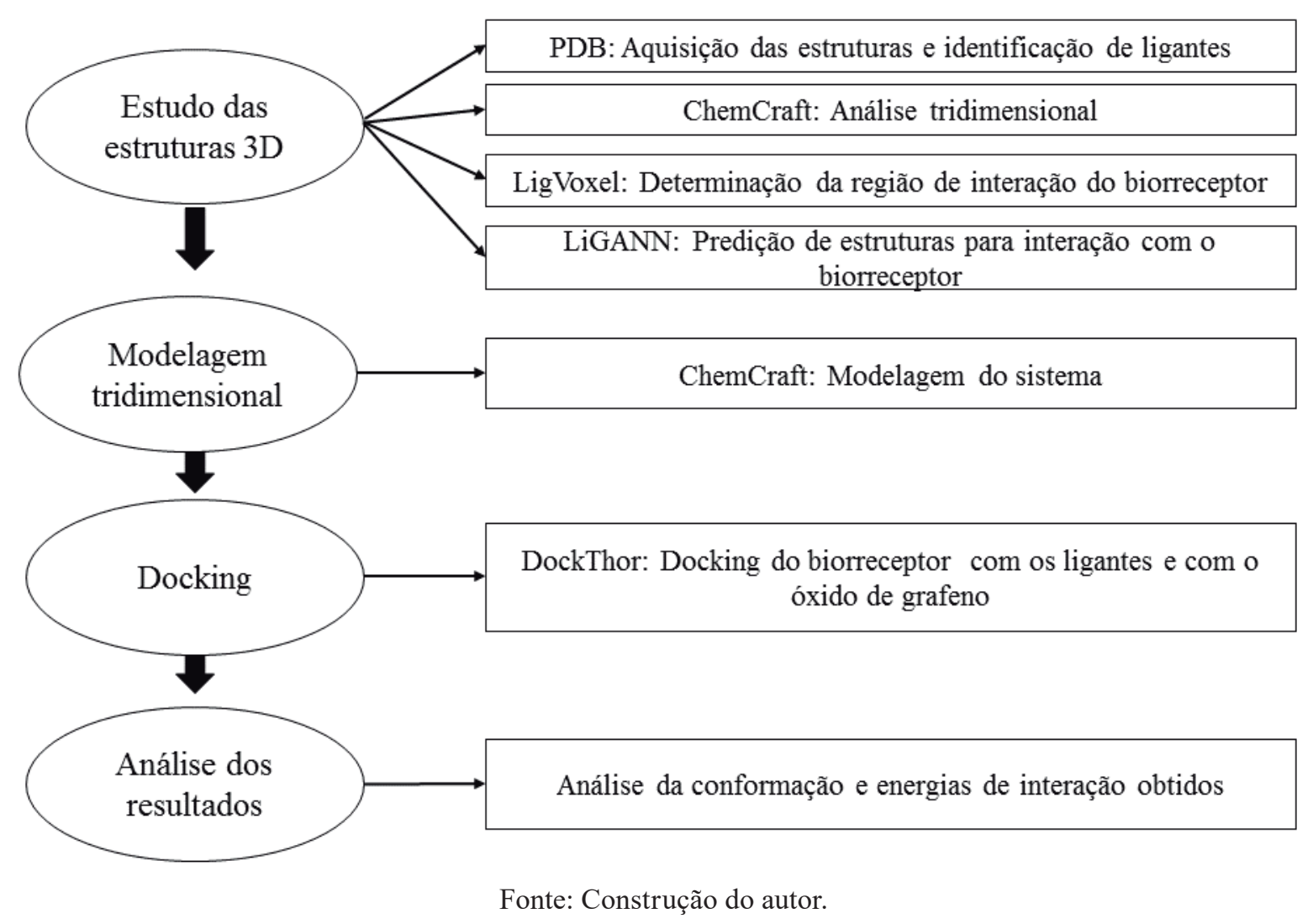

Todos os arquivos tridimensionais das estruturas, necessários para modelagem e docking, foram obtidos pelo banco de dados Protein Data Bank, e analisados por meio do software ChemCraft, onde é possível visualizar essas estruturas e manipulá-las para formar o sistema. As ferramentas LigVoxel e LiGANN do site Play Molecule, site disponível para simulações online, foram utilizadas para análise e caracterização do biorreceptor Anti-HER2. A ferramenta LigVoxel utiliza redes neurais para prever propriedades físico-químicas do ligante $3 \mathrm{D}$, e oferece as coordenadas dos locais da molécula com maior propensão à ligação com outras estruturas. Já a ferramenta $L i G A N N$ é capaz de gerar potenciais ligantes à estrutura de entrada, e assim foi analisada a semelhança de estrutura molecular dos ligantes gerados e dos ligantes BTN e EDO, cuja interação possui comprovação experimental na literatura.

O docking foi feito por meio do sistema DockThor, que permite a realização de simulações com a utilização de cofatores, de modo que se pode acrescentar diferentes ambientes de interação à simulação. Tendo em vista que o dispositivo não seria utilizado em ambientes complexos, optamos por não acrescentar cofatores às simulações. Por meio do DockThor as informações fornecidas podem ser processadas pelo supercomputador Santos Dummont do Laboratório Nacional de Ciência da Computação (LNCC). As simulações das interações entre os possíveis componentes do nanobiossensor foram feitas de acordo com as configurações mostradas na Tabela 1. 
Tabela 1 - Configurações utilizadas para docking.

\begin{tabular}{ccccc}
\hline Configuração & Simulação 1 & Simulação 2 & Simulação 3 & Simulação 4 \\
\hline Proteína & Anti-HER2 & Anti-HER2 & Anti-HER2 & Anti-HER2 \\
Ligante & BTN & EDO & GO + BTN & GO + EDO \\
\hline
\end{tabular}

A técnica de docking permite a identificação das posições de ligação que mantém o sistema estável, por meio de análise das interações intermoleculares e suas respectivas energias de interação. Dessa forma obteve-se como resultado para cada uma das simulações da tabela 1: uma imagem com a conformação tridimensional das ligações, que mostra os locais onde foi possível a interação das estruturas, quando há maximização das forças de interação e minimização da energia de interação no sistema; e uma pontuação que classifica essas posições de interação de modo que o sistema é mais estável quanto mais negativo for a energia de interação (RABELLO, 2016).

\section{RESULTADOS E DISCUSSÃO}

\section{Análise das estruturas tridimensionais}

Utilizando o software LigVoxel, foi possível determinar a região da molécula Anti-HER2 que apresenta propensão à ligação com outras estruturas. Na figura 4 observa-se a representação dessa região de interação. A partir dessa informação, é compreendido que essa região é mais propensa a receber ligantes, e a possibilidade de ligação nessa área mostra ligações estáveis.

Figura 4 - Região de docking do Anti-HER2, destacada a região com propensão à interação.

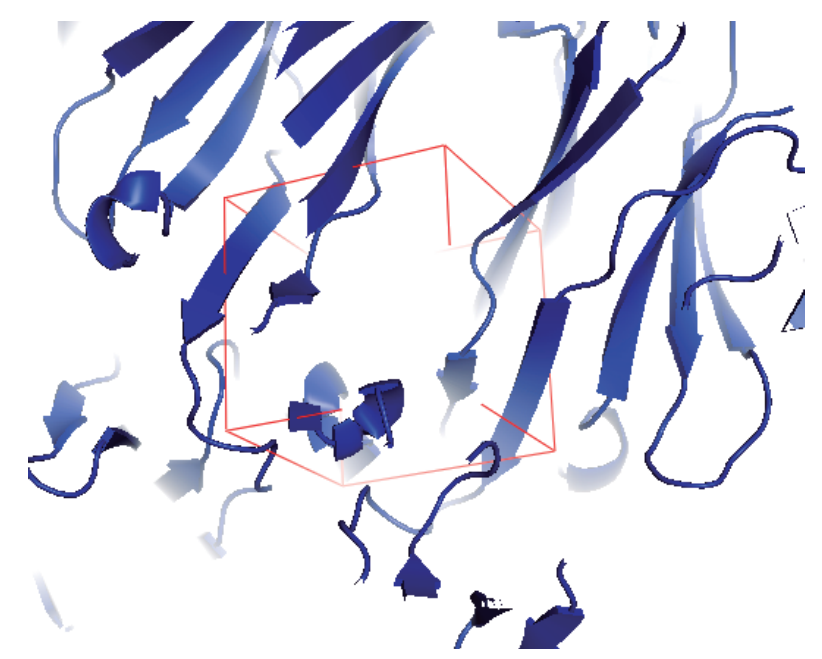

Fonte: Construção do autor.

Ao utilizar o software $L i G A N N$, foram gerados diversos potenciais ligantes para interação com a molécula Anti-HER2. Dentre elas, destacamos as estruturas apresentadas na Tabela 2, por apresentarem similaridade às estruturas BTN ou EDO. 
Assim, pode-se presumir o sucesso da interação dos ligantes BTN e EDO com o Anti-HER2. Além disso, essas interações são confirmadas pelos dados experimentais do PDB, e assim valida-se o resultado obtido pelo $L i G A N N$.

Tabela 2 - Estruturas geradas pelo LiGANN ao BTN ou ao EDO.

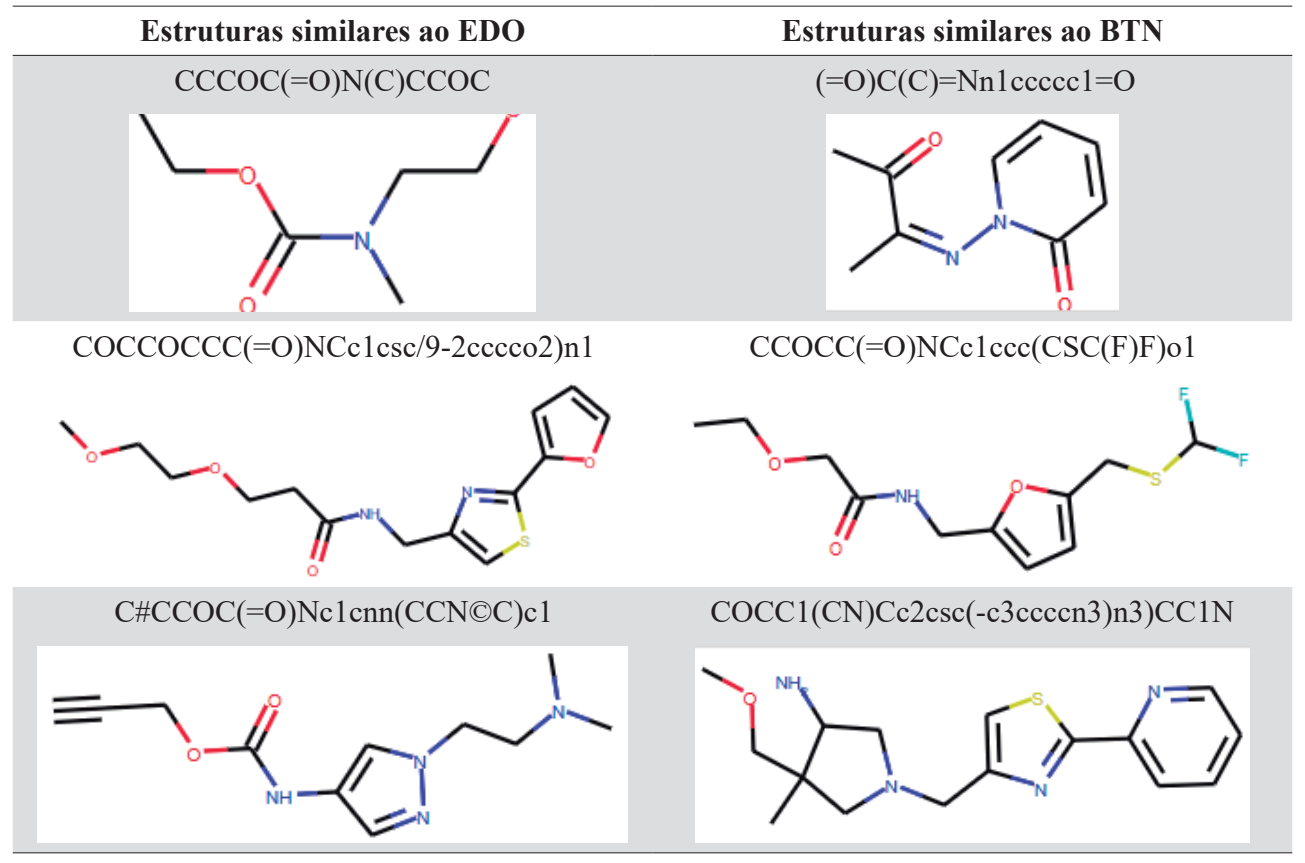

Fonte: Construção do autor.

\section{Modelagem tridimensional}

Por meio do software ChemCraft foi simulada a interação do GO com os ligantes. Na figura 5 é representada a estrutura composta pelo GO e o BTN. Enquanto a estrutura composta pelo GO e EDO é representada na figura 8.

Figura 5 - Modelagens sistema GO + BTN (5A), GO + EDO (5B).

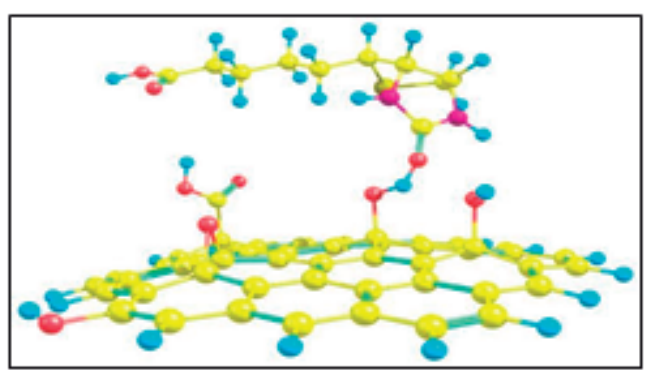

$5 \mathrm{~A}$

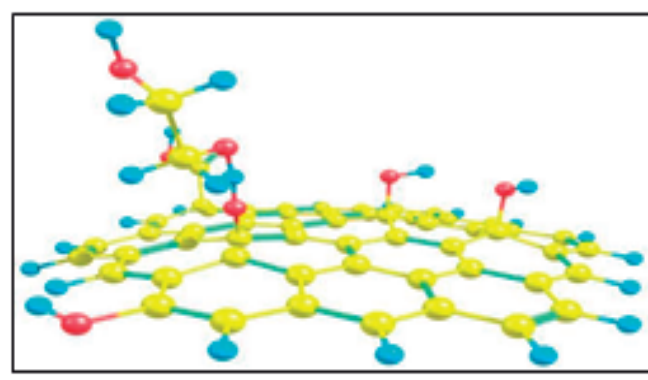

$5 \mathrm{~B}$

Fonte: Construção do autor. 


\section{DOCKING: Conformação Tridimensional}

O docking entre o Anti-HER2 e o BTN apresentou a conformação tridimensional apresentada na figura 6. Nela, pode-se observar a ausência de ligações de BTN no centro do Anti-HER2, local definido como propenso a interações, o que indica a fraca ligação entre essas estruturas.

Figura 6 - Docking do Anti-HER2 e BTN puro.

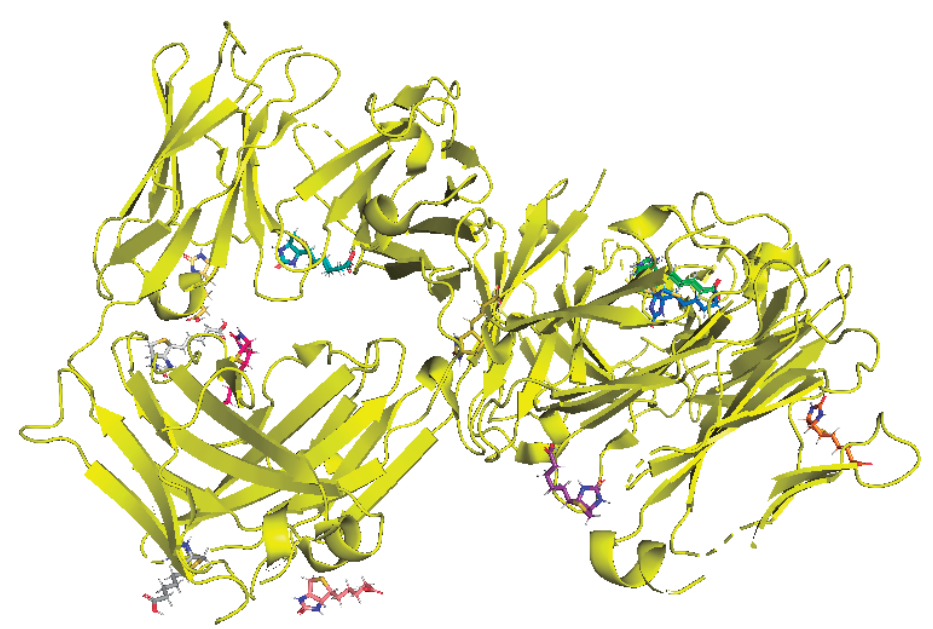

Fonte: Construção do autor.

Após, foi feito docking do sistema completo. Na figura 7 é apresentada a conformação resultante do docking entre o Anti-HER 2 e da estrutura GO + BTN. A estrutura esbranquiçada no centro da imagem é o GO + BTN, pode-se perceber que o GO impede a aproximação do BTN à estrutura do Anti-HER2.

Figura 7 - Conformação tridimensional da interação do Anti-HER2 com o BTN.

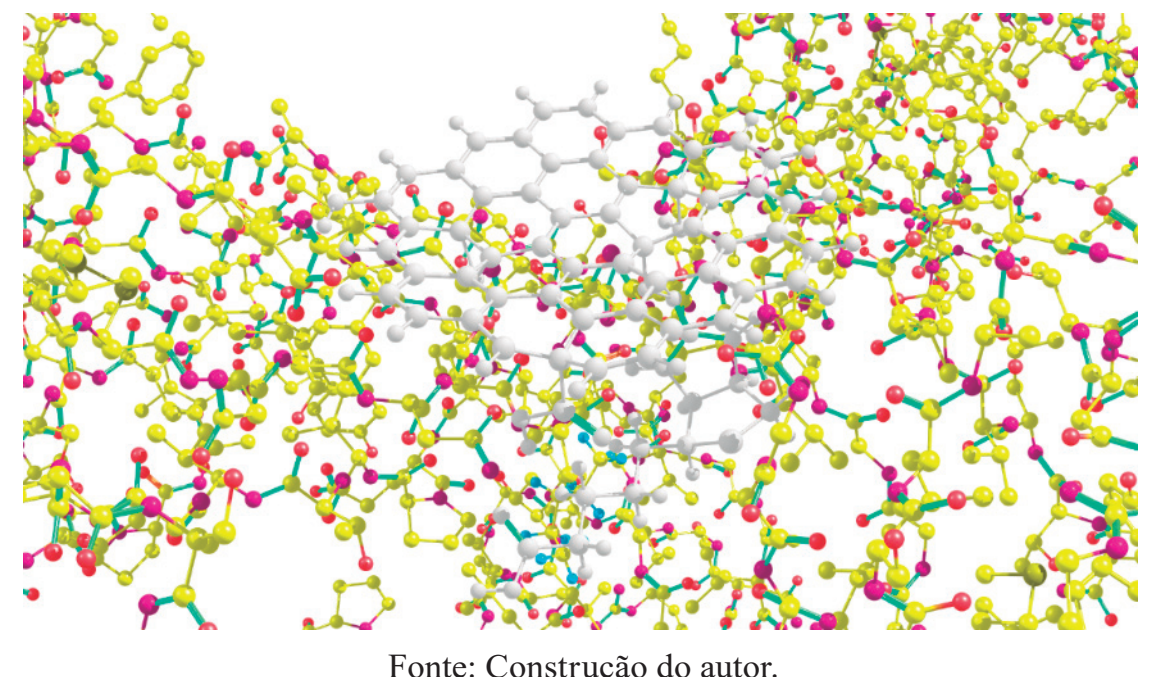

O docking entre o Anti-HER2 apresentou a conformação tridimensional mostrada na figura 8, onde pode ser visualizada a interação do EDO no centro da estrutura, região com propensão de interação do Anti-HER2. 
Figura 8 - Conformação tridimensional da interação do Anti-HER2 com o EDO.

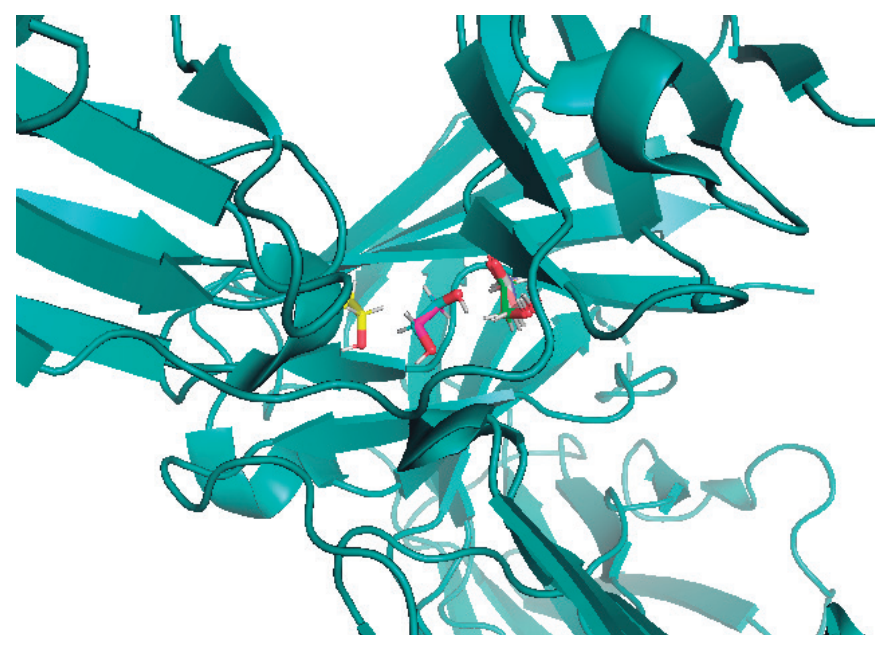

Fonte: Construção do autor.

Após analisar a interação com o ligante, foi feita a interação entre o Anti-HER2 e a estrutura composta pelo GO + EDO.

\section{DOCKING: Sistema de pontuação e energias de interação}

O docking faz a simulação de diversas posições do ligante na proteína HER2, e para facilitar a análise entre essas posições é criado um sistema de pontuação, de acordo com as energias encontradas em cada tentativa. Para favorecer a leitura e interpretação destes resultados, estes serão apresentados em tabelas que mostram as informações da interação com maior estabilidade.

Figura 9 - Conformação tridimensional da interação do Anti-HER2 com a estrutura GO + EDO.

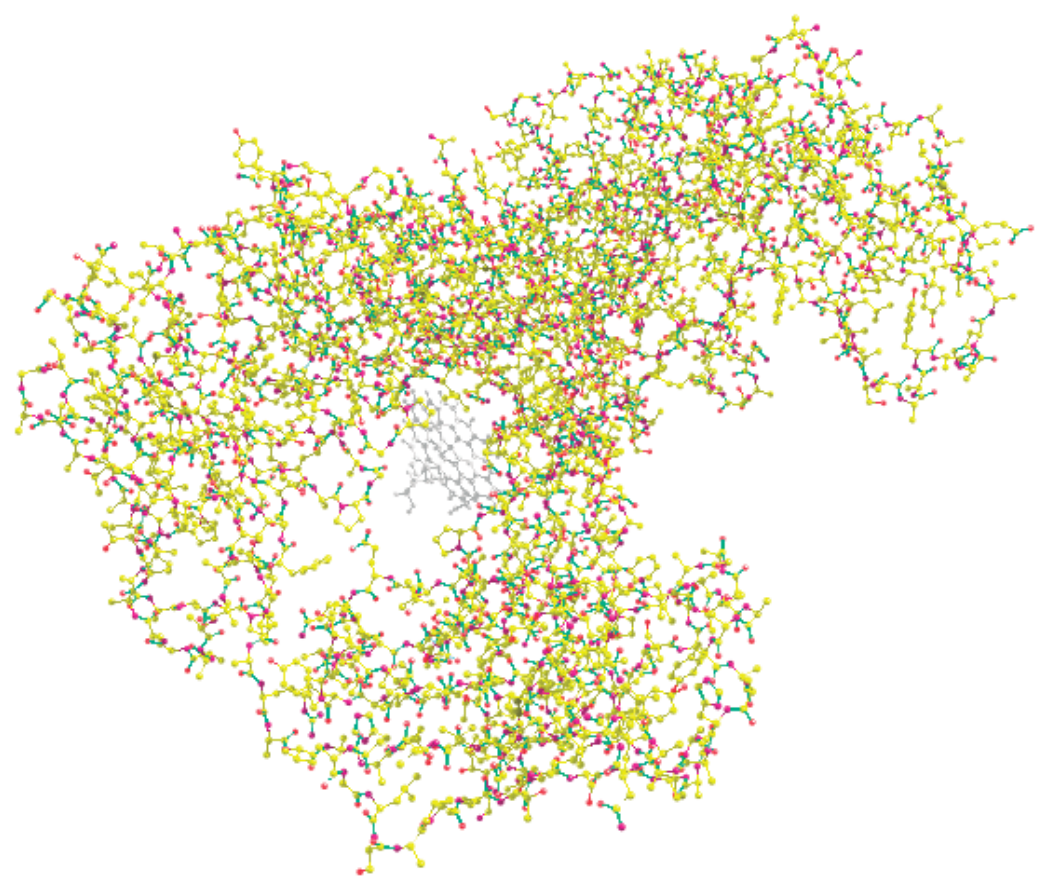

Fonte: Construção do autor. 
A interação Anti-HER2 com o BTN, representados na figura 9, demonstrou os resultados apresentados na Tabela 3. Observa-se que a energia de interação é bem baixa, o qual indica estabilidade na ligação.

Tabela 3 - Resultado do docking entre o Anti-HER2 com BTN.

\begin{tabular}{ccccccc}
\hline Nome & Pontuação & Energia Total & Energia de Interação & Forças VdW & Energia eletrostática & RMSD \\
\hline $\begin{array}{c}\text { ligand_3b } \\
\text { 523fbf1_1 }\end{array}$ & -6425 & -31974 & -23730 & 2654 & -26384 \\
\hline
\end{tabular}

A interação Anti-HER2 com o EDO obteve os resultados apresentados na tabela 4. Nessa situação a energia de interação é maior que a abordada anteriormente, no entanto continua sendo um valor que indica estabilidade do sistema.

Tabela 4 - Resultado do docking entre o Anti-HER2 com o EDO.

\begin{tabular}{ccccccc}
\hline Nome & Pontuação & Energia Total & Energia de Interação & Forças VdW & Energia eletrostática & RMSD \\
\hline ligand_1 & -5759 & -9776 & -16572 & 10876 & -27448 \\
f236cced_1 & & & & 0 \\
\hline
\end{tabular}

A interação Anti-HER2 com o complexo GO + BTN obteve os resultados apresentados na tabela 5, onde são apresentadas as duas melhores posições para interação. Ao compará-las pode-se observar que sua pontuação é similar, portanto ambas apresentam ligação estável, no entanto apresentam discrepância entre os valores de energia total.

Tabela 5 - Resultado do docking entre o Anti-HER2 com o sistema GO + BTN.

\begin{tabular}{|c|c|c|c|c|c|c|}
\hline Nome & Pontuação & Energia Total & Energia de Interação & Forças VdW & Energia eletrostática & RMSD \\
\hline $\begin{array}{c}\text { ligand_ } \\
\text { 7e2cce9500_2 }\end{array}$ & -5.682 & -5.329 & -0.286 & -0.000 & -0.286 & 0.000 \\
\hline $\begin{array}{c}\text { ligand__ } \\
\text { 7e2cce9500_5 }\end{array}$ & -5.554 & 16.899 & -0.001 & -0.000 & -0.001 & 0.000 \\
\hline
\end{tabular}

E, por fim, a interação Anti-HER2 com o complexo GO + EDO obteve os resultados apresentados na tabela 6. Destaca-se que ambas são posições de ligação que oferecem estabilidade ao sistema, devido a sua pontuação e baixa energia de interação. A diferença entre as energias de interação é mínima, no entanto, a energia total é significativamente diferente. A energia total indica a quantidade de energia necessária para recriar a interação, e por isso, a de menor valor seria preferível para análise do processo in vitro.

De acordo com a tabela 6, demonstra-se que as energias de interação resultantes das interações do biorreceptor Anti-HER2 com os ligantes são valores mais elevados, quando comparado com a mesma energia resultante das interações intermoleculares do sistema contento o GO. 
Tabela 6 - Resultado do docking entre o Anti-HER2 com o sistema GO + EDO.

\begin{tabular}{ccccccc}
\hline Nome & Pontuação & Energia Total & Energia de Interação & Forças VdW & Energia eletrostática & RMSD \\
\hline $\begin{array}{c}\text { ligand_ } \\
701 \text { e528300_4 } \\
\text { ligand_ }\end{array}$ & -5.545 & 29.932 & -0.002 & -0.000 & -0.002 & 0.000 \\
701 e528300_2 & -5.441 & 9.107 & -0.001 & -0.000 & -0.001 & 0.000 \\
\hline
\end{tabular}

\section{CONCLUSÃO}

Este trabalho teve como objetivo utilizar ferramentas de nanotecnologia computacional, para verificar a possibilidade de construção de um sistema de nanobiossensor proposto como método alternativo de diagnóstico precoce do câncer de mama. A constituição desse dispositivo baseou-se a partir do óxide de grafeno, associado as moléculas de biotina e etilenoglicol que por sua vez interagem com o Anti-HER2, elemento responsável pela detecção do alvo HER2. Conclui-se que através dos softwares Chem Craft, LiGANN, LigVoxel e Dockthor, foi perfeitamente possível a identificação, modificação, e análise das estruturas tridimensionais utilizadas, bem como a realização do docking entre estas.

Ao analisar todos os resultados compreende-se que o GO limita as possibilidades de interação dos ligantes com o biorreceptor proposto para o nanobiossensor, pelo significativo aumento do tamanho e impedimento estérico estrutural, entretanto ainda observa-se interação. A energia de interação dos sistemas rege a estabilidade do sistema resultante, enquanto a energia total informa a quantidade de energia necessária para realização da interação entre as estruturas. Os resultados mostraram que o sistema GO+BTN e Anti-HER2 é mais estável, devido à menor energia de interação intermolecular resultante.

O presente trabalho foi um estudo inicial sobre a construção de um dispositivo proposto para detecção de níveis da proteína HER2, a fim de auxiliar em pré-diagnóstico e acompanhamento dos níveis de HER2 no organismo de pacientes doentes. Adicionalmente, pretende-se que em trabalhos futuros seja analisada a interação superficial das nanoestruturas com a vizinhança atômica no Anti-HER2, por meio de simulações Ab Initio.

\section{REFERÊNCIAS}

BERMAN, H. M. et al. The Protein Data Bank. Nucleic acids research, v. 28, n. 1, p. 235-242, 2000. Disponível em: www.rcsb.org

CANTIGA, F. A. M. Biossensores salivares: estado da arte. 2015. Dissertacao de Mestardo, Instituto Superior de Ciências da Saúde Egaz MonizTese de Doutorado. Almada, Portugal, 2015. 
CARNEY, W. P. et al. Potential clinical utility of serum HER-2/neu oncoprotein concentrations in patients with breast cancer. Clinical Chemistry, v. 49, n. 10, p. 1579-1598, 2003. Disponível em: https://www.chemcraftprog.com/

DELMONICO, L.; ALVES, G.; DO AMARAL, L. F. P. A biologia do câncer de mama e testes moleculares de prognóstico. Revista Hospital Universitário Pedro Ernesto, v. 14, n. 0, p. 59-65, 2015. Disponível em: dockthor.lncc.br/v2/

GØTZSCHE, P. C.; JØRGENSEN, K. J. Screening for breast cancer with mammography Cochrane Database of Systematic Reviews. John Wiley and Sons Ltd, , 4 jun. 2013.

MINISTÉRIO DA SAÚDE. Instituto Nacional de Câncer. Disponível em: https://www.inca.gov.br/. Acesso em: 26 jun. 2019.

NETO, O. V. Nanotecnologia Computacional Primeira Escola de Verão em Computação. n. February, 2014.

PARREIRA, D. B.; LEITÃO, T. G. Nanobiossensores. INPI: Portugal, 2010.

PAUlA, G.; HORTA, B. MÉTOdOS DE MULTI-DOCKING GENERALIZAdo. [s.l: s.n.]. Disponível em: https://www.playmolecule.org/ e https://playmolecule.org/LiGANN/

RABELlO, M. M. UNIVERSIDADE FEDERAL DE PERNAMBUCO CENTRO DE BIOCIÊNCIAS Programa de Pós-Graduação em Inovação Terapêutica. Recife: [s.n.].

SALAHANDISH, R. et al. Nano-biosensor for highly sensitive detection of HER2 positive breast cancer. Biosensors and Bioelectronics, v. 117, p. 104-111, 2018.

SILVA, C. DE C. C. E. Desenvolvimento de Biossensores do Tipo Transistor de Efeito de Campo a Base de Grafeno (GraFET) Decorados com Nanopartículas de Ouro Aplicados na Detecção Ultra-sensível de Biomarcadores de Câncer de Mama. Tese de doutorado, Unicamp. São Paulo, 2015. 
